(C) 2012 IEEE. Personal use of this material is permitted. Permission from IEEE must be obtained for all other uses, in any current or future media, including reprinting/republishing this material for advertising or promotional purposes, creating new collective works, for resale or redistribution to servers or lists, or reuse of any copyrighted component of this work in other works. 


\title{
The Generalised Discrete Algebraic Riccati Equation arising in LQ optimal control problems: Part II
}

\author{
Augusto Ferrante and Lorenzo Ntogramatzidis
}

\begin{abstract}
In this paper we develop an analytic approach to the solution of a very general class of discrete finitehorizon optimal control problems. This method hinges on a new decomposition of the so-called extended symplectic pencil. Interestingly, the results established in this paper hold under assumptions that are weaker than the ones considered in the literature so far.
\end{abstract}

\section{INTRODUCTION}

The aim of this paper is to present a method to solve the most general class of finite-horizon linear-quadratic (LQ) optimal control problems in the discrete time with positive semi-definite cost index and affine constraints at the endpoints.

The approach taken in this paper is based on a parameterisation of the set of trajectories generated by the so-called extended symplectic difference equation (ESDE). The idea of solving finite-horizon LQ problems by exploiting expressions of the trajectories generated by the Hamiltonian system in the continuous time or the ESDE in the discrete time originated in the papers [2], [14] and [5] for the continuous time, and in [3] and [4] for the discrete time. In both situations, the expressions parameterising the trajectories of the Hamiltonian system and the symplectic equation hinge on particular solutions of the associated continuous/discrete algebraic Riccati equations and on the solution of the corresponding closed-loop continuous/discrete Lyapunov equation. While controllability of the given system was required in the first papers [2] [3], because both the stabilising and antistabilising solutions of the ARE were involved, in more recent times it has been shown that generalisations of the same technique are possible under the much milder assumption of sign-controllability in the continuous case [5] and modulus controllability in the discrete case, see [4]. The assumptions of sign/modulus-controllability (or stabilisability) were needed in the above-mentioned papers because the solution presented there was based on the existence of a solution of the closed-loop Lyapunov equation. In the discrete case, the other standing assumption was the regularity of the extended symplectic pencil. The goal of this paper is to propose a new approach aimed at overcoming these limitations. Indeed, in

Partially supported by the Italian Ministry for Education and Research (MIUR) under PRIN grant n. 20085FFJ2Z "New Algorithms and Applications of System Identification and Adaptive Control" and by the Australian Research Council under the grant FT120100604.

A. Ferrante is with Dipartimento di Ingegneria dell'Informazione, Università di Padova, via Gradenigo, 6/B - 35131 Padova, Italy. augustoddei.unipd. it

L. Ntogramatzidis is with the Department of Mathematics and Statistics, Curtin University, Perth (WA), Australia. L. Ntogramatzidis@curtin.edu.au this paper a direct method is developed which generalises the technique in [3] and [4] in two directions. First, we do not require the symplectic pencil to be regular, nor to have a spectrum devoid of eigenvalues on the unit circle. As such, here regular and singular problems can be tackled in a unified manner. Second, unlike the other contributions on this topic, the method presented in this paper does not involve the solvability of the closed-loop Lyapunov equation. Therefore, even the modulus controllability assumption can be dropped. The technique presented in this paper only requires a solution of the so-called generalised discrete-time algebraic Riccati equation, which may exist even when the symplectic pencil is not regular (while in this case the standard discrete algebraic Riccati equation cannot be solved). Such solution is used to derive a decomposition of the extended symplectic pencil that yields a natural parameterisation of the solutions of the symplectic difference equation. A large number of LQ problems dealt with in the literature by resorting to different - often iterative - techniques can be tackled in a unified framework and in finite, nonrecursive terms, by means of the method developed in this paper.

\section{StATEMENT OF THE PROBLEM}

Consider the linear time-invariant discrete-time system

$$
x(t+1)=A x(t)+B u(t),
$$

where, for all $t \in \mathbb{N}, x(t) \in \mathbb{R}^{n}$ is the state, $u(t) \in \mathbb{R}^{m}$ is the control input, $A \in \mathbb{R}^{n \times n}$ and $B \in \mathbb{R}^{n \times m}$. Let $T \in \mathbb{N} \backslash\{0\}$ be the length of the time horizon. Let $V_{0}, V_{T} \in \mathbb{R}^{q \times n}$ and $v \in \mathbb{R}^{q}$; consider the two-point boundary-value affine constraint

$$
V_{0} x(0)+V_{T} x(T)=v .
$$

We can consider $V \triangleq\left[\begin{array}{ll}V_{0} & V_{T}\end{array}\right]$ to be of full row rank with no loss of generality. Let $\Pi=\left[\begin{array}{cc}Q & S \\ S^{\mathrm{T}} & R\end{array}\right]=\Pi^{\mathrm{T}} \geq 0$ be a square $(n+m)$-dimensional matrix with $Q \in \mathbb{R}^{n \times n}, S \in \mathbb{R}^{n \times m}$ and $R \in \mathbb{R}^{m \times m}$; note that we do not assume the non-singularity of $R$. We denote by $\Sigma$ the Popov triple $(A, B, \Pi)$. Finally, let $H=\left[\begin{array}{ll}H_{1} & H_{2} \\ H_{2}^{\mathrm{T}} & H_{3}\end{array}\right]=H^{\mathrm{T}} \geq 0$ with $H_{1}, H_{2}, H_{3} \in \mathbb{R}^{n \times n}$ and the target states $h_{0}, h_{T} \in \mathbb{R}^{n}$.

Problem 2.1: Find $u(t), t \in\{0, \ldots, T-1\}$ and $x(t)$, $t \in\{0, \ldots, T\}$, minimising

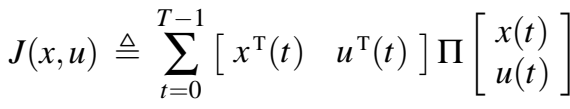

$$
\begin{aligned}
& +\left[\begin{array}{ll}
x^{\mathrm{T}}(0)-h_{0}^{\mathrm{T}} & x^{\mathrm{T}}(T)-h_{T}^{\mathrm{T}}
\end{array}\right] H\left[\begin{array}{c}
x(0)-h_{0} \\
x(T)-h_{T}
\end{array}\right]
\end{aligned}
$$

under the constraints (1-2). 
The formulation of Problem 2.1 is very general, since the cost index in (3) involves the most general type of positive semidefinite quadratic penalisation on the extreme states, and (2) represents the most general affine constraint on these states. As particular cases of Problem 2.1 we have the standard case where $x(0)$ is assigned and $x(T)$ is weighted in (3), the fixed end-point case, where the states at the end-points are sharply assigned, and the point-to-point case, where the extreme values of an output $y(t)=C x(t)$ are constrained to be equal to two assigned vectors. Further non-standard LQ problems that can be useful in practice are particular cases of Problem 2.1, see e.g., [3], [4], [5].

Lemma 2.1: [4, Lemma 3] If $u(t)$ and $x(t)$ are optimal for Problem 2.1, then $\lambda(t) \in \mathbb{R}^{n}, t \in\{0, \ldots, T\}$ and $\eta \in \mathbb{R}^{q}$ exist such that $x(t), \lambda(t), u(t)$ and $\eta$ satisfy the set of equations

$$
\begin{aligned}
& x(t+1)=A x(t)+B u(t), \quad t \in\{0, \ldots, T-1\} \\
& V\left[\begin{array}{c}
x(0) \\
x(T)
\end{array}\right]=v \\
& \lambda(t)=Q x(t)+A^{\top} \lambda(t+1)+S u(t), \quad t \in\{0, \ldots, T-1\},(6) \\
& {\left[\begin{array}{c}
-\lambda(0) \\
\lambda(T)
\end{array}\right]=H\left[\begin{array}{c}
x(0)-h_{0} \\
x(T)-h_{T}
\end{array}\right]+V^{\top} \eta} \\
& 0=S^{\top} x(t)+B^{\top} \lambda(t+1)+R u(t), \quad t \in\{0, \ldots, T-1\}
\end{aligned}
$$

Conversely, if equations (4-8) admit solutions $x(t), u(t)$, $\lambda(t), \eta$, then $x(t), u(t)$ minimise $J(x, u)$ subject to (1-2).

The variables $\lambda(t)$ in (4-8) represent the Lagrange multipliers associated with the constraint (1), [12], [10], while $\eta \in \mathbb{R}^{q}$ is the Lagrange multiplier vector associated with (2).

\section{THE GENERALISED RICCATI EQUATION AND THE EXTENDED SYMPLECTIC PENCIL}

Since in the present setting we are not assuming that $R$ is positive definite, (8) cannot be solved in $u(t)$. A convenient form in which (4), (6) and (8) can be written, that does not require inversion of $R$, is the descriptor form

$$
M p(t+1)=N p(t) \quad t \in\{0, \ldots, T-1\},
$$

where

$M \triangleq\left[\begin{array}{ccc}I_{n} & O & O \\ O & -A^{\mathrm{T}} & O \\ O & -B^{\mathrm{T}} & O\end{array}\right], \quad N \triangleq\left[\begin{array}{ccc}A & O & B \\ Q & -I_{n} & S \\ S^{\mathrm{T}} & O & R\end{array}\right], \quad p(t) \triangleq\left[\begin{array}{c}x(t) \\ \lambda(t) \\ u(t)\end{array}\right]$. The matrix pencil $N-z M$ is known as the extended symplectic pencil, [12], [10], herein denoted by $\operatorname{ESP}(\Sigma)$. We do not make the assumption of regularity of this pencil.

We now show how a solution of a generalised discrete algebraic Riccati equation can be used to obtain a decomposition of $\operatorname{ESP}(\Sigma)$ that can be used to solve Problem 2.1. In particular, we will exploit the solutions of the following constrained matrix equation

$$
\begin{gathered}
X=A^{\mathrm{T}} X A-\left(A^{\mathrm{T}} X B+S\right)\left(R+B^{\mathrm{T}} X B\right)^{-1}\left(B^{\mathrm{T}} X A+S^{\mathrm{T}}\right)+Q, \\
\operatorname{ker}\left(R+B^{\mathrm{T}} X B\right) \subseteq \operatorname{ker}\left(A^{\mathrm{T}} X B+S\right),
\end{gathered}
$$

where the matrix inverse that appears in the standard discrete algebraic Riccati equation (DARE) has been replaced by the Moore-Penrose pseudo-inverse. Eq. (10) is known in the literature as the generalised discrete-time algebraic Riccati equation $\operatorname{GDARE}(\Sigma)$, [15], [9]. $\operatorname{GDARE}(\Sigma)$ with the additional constraint given by (11) is sometimes referred to as constrained generalised discrete-time algebraic Riccati equation CGDARE $(\Sigma)$. Clearly (10) constitutes a generalisation of the classic $\operatorname{DARE}(\Sigma)$, in the sense that any solution of $\operatorname{DARE}(\Sigma)$ is also a solution of $\operatorname{GDARE}(\Sigma)$ - and therefore also of CGDARE $(\Sigma)$ - but the converse is not true in general.

Since as aforementioned the Popov matrix $\Pi$ is assumed to be symmetric and positive semidefinite, we can consider a standard factorisation of the form $\Pi=\left[\begin{array}{l}C^{\mathrm{T}} \\ D^{\mathrm{T}}\end{array}\right]\left[\begin{array}{ll}C & D\end{array}\right]$, where $Q=C^{\mathrm{T}} C, S=C^{\mathrm{T}} D$ and $R=D^{\mathrm{T}} D$. We now introduce some notation that will be used throughout the paper. First, to any matrix $X=X^{\mathrm{T}} \in \mathbb{R}^{n \times n}$ we associate the following matrices:

$S_{X} \triangleq A^{\mathrm{T}} X B+S, \quad R_{X} \triangleq R+B^{\mathrm{T}} X B, \quad G_{X} \triangleq I_{m}-R_{X}^{\dagger} R_{X}$,

$K_{X} \triangleq R_{X}^{\dagger} S_{X}^{\mathrm{T}}, \quad A_{X} \triangleq A-B K_{X}, \quad C_{X} \triangleq C-D R_{X}^{\dagger} S_{X}^{\mathrm{T}}$.

The term $R_{X}^{\dagger} R_{X}$ is the orthogonal projector that projects onto $\operatorname{im} R_{X}^{\dagger}=\operatorname{im} R_{X}$ so that $G_{X}$ is the orthogonal projector that projects onto $\operatorname{ker} R_{X}$. Hence, $\operatorname{ker} R_{X}=\operatorname{im} G_{X}$.

Let $\mathscr{R}_{X}$ denote the reachable subspace associated with the pair $\left(A, B G_{X}\right)$, in symbols $\mathscr{R}_{X} \triangleq$ $\operatorname{im}\left[\begin{array}{lllll}B G_{X} & A_{X} B G_{X} & A_{X}^{2} B G_{X} & \ldots & A_{X}^{n-1} B G_{X}\end{array}\right]$.

The following results were proved in [6, Lemma 4.1, Lemma 4.2, Theorem 4.2].

Lemma 3.1: Let $X=X^{\mathrm{T}}$ be a solution of $\operatorname{CGDARE}(\Sigma)$. Then,

(i) $\mathscr{R}_{X} \subseteq \operatorname{ker} C_{X}$;

(ii) $\operatorname{ker} R_{X}=\operatorname{ker}(X B) \cap \operatorname{ker} R$;

(iii) $\mathscr{R}_{X}$ is the reachability subspace on the output-nulling subspace $\operatorname{ker} X$.

We also have the following results, see [6, Theorems 4.34.4].

Lemma 3.2: The subspaces $\operatorname{ker} R_{X}$ and $\mathscr{R}_{X}$, and the restriction $\left.A_{X}\right|_{\mathscr{R}_{X}}$ do not depend on the solution $X=X^{\mathrm{T}}$ of $\operatorname{CGDARE}(\Sigma)$.

The following result adapts [8, Lemma 2.5] to the case when the matrix pencil $N-z M$ may be singular.

Lemma 3.3: Let $X=X^{\mathrm{T}}$ be a solution of $\operatorname{CGDARE}(\Sigma)$. Then, $U_{X}, V_{X} \in \mathbb{R}^{2 n+m}$ exist such that

$$
U_{X}(N-z M) V_{X}=\left[\begin{array}{ccc}
A_{X}-z I_{n} & O & B \\
O & I_{n}-z A_{X}^{\mathrm{T}} & O \\
O & -z B^{\mathrm{T}} & R_{X}
\end{array}\right] .
$$

Proof: The statement follows by using

$$
U_{X} \triangleq\left[\begin{array}{ccc}
I_{n} & O & O \\
A_{X}^{\mathrm{T}} X & I_{n} & -K_{X}^{\mathrm{T}} \\
B^{\mathrm{T}} X & O & I_{m}
\end{array}\right], V_{X} \triangleq\left[\begin{array}{ccc}
I_{n} & O & O \\
X & -I_{n} & O \\
-K_{X} & O & I_{m}
\end{array}\right]
$$

If $X$ is a solution of $\operatorname{CGDARE}(\Sigma)$, from the triangular structure in (14) we have

$$
\operatorname{det}(N-z M)=\operatorname{det}\left(A_{X}-z I_{n}\right) \cdot \operatorname{det}\left(I_{n}-z A_{X}^{\mathrm{T}}\right) \cdot \operatorname{det} R_{X} \cdot
$$

When $R_{X}$ is non-singular (i.e. $X$ is a solution of $\operatorname{DARE}(\Sigma)$ ), the dynamics represented by this matrix pencil are decomposed into a part governed by the generalised eigenstructure 
of $A_{X}-z I_{n}$, a part governed by the finite generalised eigenstructure of $I_{n}-z A_{X}^{\mathrm{T}}$, and a part which corresponds to the dynamics of the eigenvalues at infinity. When $X$ is a solution of $\operatorname{DARE}(\Sigma)$, the generalised eigenvalues ${ }^{1}$ of $N-M z$ are given by the eigenvalues of $A_{X}$, the reciprocal of the non-zero eigenvalues of $A_{X}$, and a generalised eigenvalues at infinity whose algebraic multiplicity is equal to $m$ plus the algebraic multiplicity of the eigenvalue of $A_{X}$ at the origin, and we have

$$
\sigma(N-z M)=\sigma\left(A_{X}-z I_{n}\right) \cup \sigma\left(\left[\begin{array}{cc}
I_{n}-z A_{X}^{\mathrm{T}} & O \\
-z B^{\mathrm{T}} & R_{X}
\end{array}\right]\right) .
$$

When the matrix $R_{X}$ is singular, (15) still holds but provides no information as in this case $\operatorname{det} R_{X}=0$, while (16) is no longer true. We show this fact with a simple example.

Theorem 3.1: Let CGDARE $(\Sigma)$ admit a solution $X=X^{\mathrm{T}}$. Two matrices $\hat{U}_{X}$ and $\hat{V}_{X}$ exist such that

$$
\begin{aligned}
& \hat{U}_{X}(N-z M) \hat{V}_{X} \\
& =\left[\begin{array}{cccccc}
A_{X, 11}-z I_{r} & B_{21} & O & A_{X, 12} & O & B_{11} \\
\hline O & O & I_{r}-z A_{X, 11}^{\mathrm{T}} & O & O & O \\
O & O & -z B_{21}^{\mathrm{T}} & O & O & O \\
\hline O & O & O & A_{X, 22}-z I_{n-r} & O & B_{12} \\
O & O & -z A_{X, 12}^{\mathrm{T}} & O & I_{n-r}-z A_{X, 22}^{\mathrm{T}} & O \\
O & O & -z B_{11}^{\mathrm{T}} & O & -z B_{12}^{\mathrm{T}} & R_{X, 0}
\end{array}\right] .
\end{aligned}
$$

where the pair $\left(A_{X, 11}, B_{21}\right)$ is reachable and $R_{X, 0}$ is invertible. Moreover, the matrix pencil $P_{1}(z) \triangleq$ $\left[\begin{array}{ccc}A_{X, 22}-z I_{n-r} & O & B_{12} \\ O & I_{n-r}-z A_{X}^{\mathrm{T}} & O \\ O & -z B_{12}^{\mathrm{T}}, & R_{X, 0}\end{array}\right]$ in (17) is regular, and the generalised eigenvalues of the pencil $N-z M$ are the generalised eigenvalues of $P_{1}(z)$.

Proof: The statement can be proved by considering two changes of coordinates: one is $\hat{T} \triangleq \operatorname{diag}\left(I_{n}, I_{n}, T\right)$, where $T=\left[\begin{array}{ll}T_{1} & T_{2}\end{array}\right]$ is an orthogonal transformation in the input space with $\operatorname{im} T_{1}=\operatorname{im} R_{X}$ and $\operatorname{im} T_{2}=\operatorname{im} G_{X}=\operatorname{ker} R_{X}$, so that $T^{\mathrm{T}} R_{X} T=\operatorname{diag}\left\{R_{X, 0}, O\right\}$, where $R_{X, 0}$ is invertible. The second is given by $\hat{U}=\operatorname{diag}\left\{U, U, I_{m_{1}}, I_{m_{2}}\right\}$, where $U=\left[\begin{array}{ll}U_{1} & U_{2}\end{array}\right]$ is such that $\operatorname{im} U_{1}$ is the reachable subspace associated with the pair $\left(A_{X}, B_{2}\right)$, which coincides with the subspace $\mathscr{R}_{X}$, so that

$$
\begin{aligned}
U^{-1} A_{X} U & =\left[\begin{array}{cc}
A_{X, 11} & A_{X, 12} \\
O & A_{X, 22}
\end{array}\right], \\
U^{-1} B_{2} & =\left[\begin{array}{c}
B_{21} \\
O
\end{array}\right], \quad U^{-1} B_{1}=\left[\begin{array}{l}
B_{11} \\
B_{12}
\end{array}\right] .
\end{aligned}
$$

Thus, defining the matrices $B_{1} \triangleq B T_{1}$ and $B_{2} \triangleq B T_{2}$, the statement follows by simply reordering the blocks via two unimodular matrices.

From these considerations, it turns out that the eigenvalues of $A_{X}$ restricted to $\mathscr{R}_{X}$ do not appear as generalised eigenvalues of $\operatorname{ESP}(\Sigma)$, whereas the eigenvalues of the map induced by $A_{X}$ in the quotient space $\mathbb{R}^{n} / \mathscr{R}_{X}$ - along with the reciprocals of those that are different from zero - are generalised eigenvalues of $\operatorname{ESP}(\Sigma)$.

\footnotetext{
${ }^{1}$ Recall that a generalised eigenvalue of a matrix pencil $N-z M$ is a value of $z \in \mathbb{C}$ for which the rank of the matrix pencil $N-z M$ is lower than its normal rank.
}

Example 3.1: Consider the following matrices

$$
\begin{aligned}
& A=\left[\begin{array}{cccc}
2 & 0 & 0 & 0 \\
-1 & 0 & 0 & -3 \\
0 & 0 & 0 & 0 \\
0 & 0 & 0 & 1
\end{array}\right], \quad B=\left[\begin{array}{cc}
1 & 0 \\
0 & 3 \\
2 & 0 \\
0 & -2
\end{array}\right], \\
& C=\left[\begin{array}{llll}
0 & 0 & 0 & 1 \\
0 & 0 & 0 & 0
\end{array}\right], \quad D=\left[\begin{array}{cc}
0 & 0 \\
0 & 1
\end{array}\right],
\end{aligned}
$$

with $Q=C^{\mathrm{T}} C=\operatorname{diag}\{0,0,0,1\}, S=C^{\mathrm{T}} D=0, R=D^{\mathrm{T}} D=$ $\operatorname{diag}\{0,1\}$. In this case, $\operatorname{DARE}(\Sigma)$ has no solutions. A solution of $\operatorname{CGDARE}(\Sigma)$ is $X_{ \pm}=\operatorname{diag}\left\{0,0,0, \frac{1 \pm \sqrt{2}}{2}\right\}$. Let us consider the positive semidefinite solution $X=X_{+}$. The gain matrix is $K_{X}=\left[\begin{array}{cccc}0 & 0 & 0 & 0 \\ 0 & 0 & 0 & 1-\sqrt{2}\end{array}\right]$ and the closed-loop matrix is

$$
A_{X}=\left[\begin{array}{cccc}
2 & 0 & 0 & 0 \\
-1 & 0 & 0 & 3(\sqrt{2}-2) \\
0 & 0 & 0 & 0 \\
0 & 0 & 0 & 3-2 \sqrt{2}
\end{array}\right] .
$$

A basis matrix of the subspace $\operatorname{ker} X$ is $\left[\begin{array}{c}I_{3} \\ O\end{array}\right]$, and is outputnulling. A simple computation shows that $\left[\begin{array}{llll}1 & 0 & 2 & 0\end{array}\right]^{\mathrm{T}}$ is a basis matrix for $\operatorname{ker} X \cap B \operatorname{ker} D$. The reachability subspace on $\operatorname{ker} X$ can be computed as the smallest $A_{X}$-invariant subspace containing $\operatorname{ker} X \cap B \operatorname{ker} D$. It is given by

$$
\mathscr{R}_{X}=\operatorname{im}\left[\begin{array}{cc}
17 & 0 \\
-8 & 1 \\
2 & 4 \\
0 & 0
\end{array}\right]
$$

In order to find the fixed internal eigenvalues of $\operatorname{ker} X$ we choose a change of basis $T=\left[\begin{array}{lll}T_{1} & T_{2} & T_{3}\end{array}\right]$ where im $T_{1}=\mathscr{R}_{X}$, $\operatorname{im}\left[\begin{array}{ll}T_{1} & T_{2}\end{array}\right]=\operatorname{ker} X$ and $T_{3}$ is such that $T$ is invertible. For example, let us choose

$T_{1}=\left[\begin{array}{cc}17 & 0 \\ -8 & 1 \\ 2 & 4 \\ 0 & 0\end{array}\right], \quad T_{2}=\left[\begin{array}{l}0 \\ 0 \\ 1 \\ 0\end{array}\right], \quad T_{3}=\left[\begin{array}{l}0 \\ 0 \\ 0 \\ 1\end{array}\right]$,

so that

$$
T^{-1} A_{X} T=\left[\begin{array}{cc|c||c}
2 & 0 & 0 & 0 \\
-1 & 0 & 0 & 3(\sqrt{2}-2) \\
\hline 0 & 0 & 0 & 12(2-\sqrt{2}) \\
\hline \hline 0 & 0 & 0 & 3-2 \sqrt{2}
\end{array}\right] .
$$

The eigenvalues 2 and 0 in the top left block are eigenvalues of $A_{X}$ but are not generalised eigenvalues of the extended symplectic pencil. The eigenvalue of $A_{X}$ induced in the quotient space $\operatorname{ker} X / \mathscr{R}_{X}$ is equal to zero, and it appears as generalised eigenvalue of the extended symplectic pencil. The eigenvalue of $A_{X}$ induced in the quotient space $\mathbb{R} / \operatorname{ker} X$ is equal to $3-2 \sqrt{2}$, and it appears as generalised eigenvalue of the extended symplectic pencil along with its reciprocal $3+2 \sqrt{2}$. In fact, these values are uncontrollable eigenvalues of the pair $\left(A_{X}, B_{2}\right)$. First, we compute $R_{X}$ and $G_{X}$ :

$R_{X}=\left[\begin{array}{cc}0 & 0 \\ 0 & 3+2 \sqrt{2}\end{array}\right], \quad G_{X}=\left[\begin{array}{ll}1 & 0 \\ 0 & 0\end{array}\right], \quad B G_{X}=\left[\begin{array}{ll}1 & 0 \\ 0 & 0 \\ 2 & 0 \\ 0 & 0\end{array}\right]$. 
The reachability subspace of the pair $\left(A_{X}, B G_{X}\right)$ coincides with $\mathscr{R}_{X}$. We perform the Kalman reachability canonical decomposition using $H=\left[\begin{array}{ll}H_{1} & H_{2}\end{array}\right]$ such that im $H_{1}=\mathscr{R}_{X}$. We take e.g.

$$
H_{1}=\left[\begin{array}{cc}
1 & 8 \\
0 & -5 \\
2 & -4 \\
0 & 0
\end{array}\right], \quad H_{2}=\left[\begin{array}{ll}
0 & 0 \\
1 & 0 \\
0 & 0 \\
0 & 1
\end{array}\right],
$$

we get

$H^{-1} A_{X} H=\left[\begin{array}{cc|cc}\frac{2}{5} & \frac{16}{5} & 0 & 0 \\ \frac{1}{5} & \frac{8}{5} & 0 & 0 \\ \hline 0 & 0 & 0 & 3(\sqrt{2}-2) \\ 0 & 0 & 0 & 3-2 \sqrt{2}\end{array}\right], \quad H^{-1}\left[\begin{array}{ll}B_{1} & B_{2}\end{array}\right]=\left[\begin{array}{c|c}0 & 1 \\ 0 & 0 \\ \hline 3 & 0 \\ -2 & 0\end{array}\right]$.

Hence, zero and $3-2 \sqrt{2}$ are both generalised eigenvalues of the extended symplectic pencil $N-z M$, and therefore so is $1 /(3-2 \sqrt{2})$. The multiplicity of the eigenvalue at infinity is equal to the multiplicity of the eigenvalue at zero of

$$
\left[\begin{array}{cc|c}
0 & 0 & 0 \\
3(\sqrt{2}-2) & 3-2 \sqrt{2} & 0 \\
\hline 0 & 0 & 3+2 \sqrt{2}
\end{array}\right] .
$$

Notice that in (19), the eigenvalues $\{0,2\}$ of $A_{X}$ restricted to $\mathscr{R}_{X}$ do not depend on $X$.

\section{SOlution OF THE LQ PROBlem}

In the basis constructed in the previous section, (9) can be written for $t \in\{0, \ldots, T-1\}$ as

$$
\begin{aligned}
x_{1}(t+1)= & A_{X, 11} x_{1}(t)+B_{21} u_{1}(t) \\
& +A_{X, 12} x_{2}(t)+B_{11} u_{2}(t), \\
\lambda_{1}(t)= & A_{X, 11}^{\mathrm{T}} \lambda_{1}(t+1), \\
0= & -B_{21}^{\mathrm{T}} \lambda_{1}(t+1), \\
x_{2}(t+1)= & A_{X, 22} x_{2}(t)+B_{12} u_{2}(t), \\
\lambda_{2}(t)= & A_{X, 22}^{\mathrm{T}} \lambda_{2}(t+1)+A_{X, 12}^{\mathrm{T}} \lambda_{1}(t+1), \\
u_{2}(t)= & R_{X, 0}^{-1} B_{12}^{\mathrm{T}} \lambda_{2}(t+1)+R_{X, 0}^{-1} B_{11}^{\mathrm{T}} \lambda_{1}(t+1) .
\end{aligned}
$$

Since by construction the pair $\left(A_{X, 11}, B_{21}\right)$ is reachable, $\operatorname{ker}\left[\begin{array}{c}A_{X, 11}^{\mathrm{T}} \\ B_{21}^{\mathrm{T}}\end{array}\right]=\{0\}$, which means $(21-22)$ yield $\lambda_{1}(t)=0$ for all $t \in\{0, \ldots, T-1\}$. This implies that (24-25) can be simplified as

$$
\begin{aligned}
& \lambda_{2}(t)=A_{X, 22}^{\mathrm{T}} \lambda_{2}(t+1), \\
& u_{2}(t)=R_{X, 0}^{-1} B_{12}^{\mathrm{T}} \lambda_{2}(t+1) .
\end{aligned}
$$

It is clear at this point that we can parameterise all the trajectories generated by the difference equations (23), (26) and (27) in terms of $x_{2}(0)$ and $\lambda_{2}(T)$. Indeed, (26) leads to

$$
\lambda_{2}(t)=\left(A_{X, 22}^{\mathrm{T}}\right)^{T-t} \lambda_{2}(T) \quad \forall t \in\{0, \ldots, T\} .
$$

This expression can be plugged into (27) and leads

$$
u_{2}(t)=R_{X, 0}^{-1} B_{12}^{\mathrm{T}}\left(A_{X, 22}^{\mathrm{T}}\right)^{T-t-1} \lambda_{2}(T) .
$$

Plugging (28) and (29) into (23) gives

$$
\begin{aligned}
x_{2}(t)= & A_{X, 22}^{t} x_{2}(0) \\
& +\sum_{j=0}^{t-1} A_{X, 22}^{t-j-1} B_{12} R_{X, 0}^{-1} B_{12}^{\mathrm{T}}\left(A_{X, 22}^{\mathrm{T}}\right)^{T-j-1} \lambda_{2}(T) .
\end{aligned}
$$

Note that $x_{2}(T)=A_{X, 22}^{T} x_{2}(0)-P \lambda_{2}(T)$, where

$$
P \triangleq \sum_{j=0}^{T-1} A_{X, 22}^{T-j-1} B_{12} R_{X, 0}^{-1} B_{12}^{\mathrm{T}}\left(A_{X, 22}^{\mathrm{T}}\right)^{T-j-1}
$$

It is easy to see that matrix $P$ can be re-written as $P=$ $\sum_{j=0}^{T-1} A_{X, 22}^{j} B_{12} R_{X, 0}^{-1} B_{12}^{\mathrm{T}}\left(A_{X, 22}^{\mathrm{T}}\right)^{j}$. Therefore, $P$ satisfies the discrete Lyapunov equation

$P=A_{X, 22} P A_{X, 22}^{\mathrm{T}}-A_{X, 22}^{T} B_{12} R_{X, 0}^{-1} B_{12}^{\mathrm{T}}\left(A_{X, 22}^{\top}\right)^{T}+B_{12} R_{X, 0}^{-1} B_{12}^{\mathrm{T}}$.

If $A_{X, 22}$ has unmixed spectrum, this equation can be used to determine $P$ instead of computing the sum in (31). At this point we can solve (20), which can be written as

$$
x_{1}(t+1)=A_{X, 11} x_{1}(t)+B_{21} u_{1}(t)+\xi(t),
$$

where $\xi(t)=A_{X, 12} x_{2}(t)+B_{11} u_{2}(t)$. Using (30) and (29) we find

$$
\begin{aligned}
\xi(t)= & A_{X, 12} A_{X, 22}^{t} x_{2}(0)+\left(B_{11} R_{X, 0}^{-1} B_{12}^{\mathrm{T}}\left(A_{X, 22}^{\mathrm{T}}\right)^{T-t-1}\right. \\
& \left.+A_{X, 12} \sum_{j=0}^{t-1} A_{X, 22}^{t-j-1} B_{12} R_{X, 0}^{-1} B_{12}^{\mathrm{T}}\left(A_{X, 22}^{\mathrm{T}}\right)^{T-j-1}\right) \lambda_{2}(T) .
\end{aligned}
$$

Let $R_{1}=\left[B_{21}\left|A_{X, 11} B_{21}\right| A_{X, 11}^{2} B_{21}|\cdots| A_{X, 11}^{T-1} B_{21}\right]$ and $R_{2}=\left[I\left|A_{X, 11}\right| A_{X, 11}^{2}|\cdots| A_{X, 11}^{T-1}\right]$. Then, we can write $x_{1}(T)=A_{X, 11}^{T} x_{1}(0)+R_{2} \Xi+R_{1} U_{1}$ where $\Xi \triangleq\left[\begin{array}{c}\xi(T-1) \\ \vdots \\ \xi(0)\end{array}\right]$ and $U_{1} \triangleq\left[\begin{array}{c}u_{1}(T:-1) \\ \vdots \\ u_{1}(0)\end{array}\right]$. We assume that $T$ is greater than the controllability index of the pair $\left(A_{X, 11}, B_{21}\right)$. All the solutions of this equation are parameterised by

$$
U_{1}=R_{1}^{\dagger}\left(x_{1}(T)-A_{X, 11}^{T} x_{1}(0)-R_{2} \Xi\right)+\left(I-R_{1}^{\dagger} R_{1}\right) v_{1} .
$$

where $v_{1}$ is arbitrary.

\section{A. Boundary conditions}

Consider the change of coordinates given by the matrix $U=\left[\begin{array}{ll}U_{1} & U_{2}\end{array}\right]$, where $\operatorname{im} U_{1}$ is the reachable subspace of the pair $\left(A_{X}, B G_{X}\right)$. Let $\left[\begin{array}{l}x_{1}(t) \\ x_{2}(t)\end{array}\right]=U^{-1} x(t)$ be the coordinates of the state in the basis induced by $U$, partitioned conformably with $U$. The state, co-state and transversality equations can be written again as in (4), (6) and (8), where $A, B, Q, S, V$, $H, h_{0}$ and $h_{T}$ are replaced by $U^{-1} A U, U^{-1} B, U^{\mathrm{T}} Q U, U^{\mathrm{T}} S$, $V\left[\begin{array}{ll}U & O \\ O & U\end{array}\right],\left[\begin{array}{ll}U & O \\ O & U\end{array}\right]^{\mathrm{T}} H\left[\begin{array}{ll}U & O \\ O & U\end{array}\right], U^{-1} h_{0}$ and $U^{-1} h_{T}$, respectively. We can now write (5) and (7) with respect to this basis. We can eliminate the multiplier $\eta$ from (7) by premultiplying both sides of this equation by a basis $K_{V}$ of $\operatorname{ker} V$ :

$K_{V}^{\mathrm{T}} H\left[\begin{array}{l}x(0) \\ x(T)\end{array}\right]+K_{V}^{\mathrm{T}}\left[\begin{array}{cc}I & O \\ O & -I\end{array}\right]\left[\begin{array}{l}\lambda(0) \\ \lambda(T)\end{array}\right]=K_{V}^{\mathrm{T}} H\left[\begin{array}{l}h_{0} \\ h_{T}\end{array}\right]$.

In this way, (5) and (34) can be written together as a set of $2 n$ linear equations in $x(0), x(T), \lambda(0)$ and $\lambda(T)$. However, 
in (31) the component $x_{2}(T)$ is expressed as a linear function of $x_{2}(0)$ and $\lambda_{2}(T)$, and $\lambda_{2}(0)$ can be expressed as a linear function in $\lambda_{2}(T)$ by (28). Finally we know that $\lambda_{1}(t)$ must be identically zero, so that $\lambda_{1}(0)=\lambda_{1}(T)=0$. Therefore, in this basis (5) and (7) can be expressed as a single linear equation of the form

$$
F x=g, \quad \text { where } \quad x=\left[\begin{array}{c}
x_{1}(0) \\
x_{1}(T) \\
x_{2}(0) \\
\lambda_{2}(T)
\end{array}\right] .
$$

We have just proved the following result.

Theorem 4.1: Problem 2.1 admits solutions if and only if (35) does. For any solution $x=$ $\left[\begin{array}{llll}x_{1}^{\mathrm{T}}(0) & x_{1}^{\mathrm{T}}(T) & x_{2}^{\mathrm{T}}(0) & \lambda_{2}^{\mathrm{T}}(T)\end{array}\right]^{\mathrm{T}} \quad$ we get an optimal initial state $x(0)=\left[\begin{array}{l}x_{1}(0) \\ x_{2}(0)\end{array}\right]$ and a class of optimal controls parameterised by (29) and (33). The solutions obtained in this way are all the solutions of Problem 2.1.

\section{AN ILlUSTRATIVE EXAMPLE}

Consider a finite-horizon LQ problem in the time interval $\{0, \ldots, T\}$, involving the matrices

$A=\left[\begin{array}{ll}1 & 1 \\ 0 & 1\end{array}\right], \quad B=\left[\begin{array}{ll}2 & 0 \\ 1 & 1\end{array}\right], \quad C=\left[\begin{array}{ll}0 & 1\end{array}\right], \quad D=\left[\begin{array}{ll}0 & 0\end{array}\right]$.

Let $Q=C^{\mathrm{T}} C, S=C^{\mathrm{T}} D$ and $R=D^{\mathrm{T}} D$. The extended symplectic pencil in this case is not regular. As such, $\operatorname{DARE}(\Sigma)$ in this case does not admit solutions. On the other hand, in this case $\operatorname{GDARE}(\Sigma)$ admits the solution $X=\operatorname{diag}\{0,1\}$, that can be computed for example by resorting to the algorithm proposed in [1]. In this case, $R_{X}=R+B^{\mathrm{T}} X B=\left[\begin{array}{ll}1 & 1 \\ 1 & 1\end{array}\right]$, and the corresponding closed-loop matrix is $A_{X}=\operatorname{diag}\{1,0\}$. Observe that the spectrum of $A_{X}$ is not unmixed. For this system we have $G_{X}=I_{m}-R_{X}^{\dagger} R_{X}=\frac{1}{2}\left[\begin{array}{cc}1 & -1 \\ -1 & 1\end{array}\right]$. Since $X$ satisfies (11), being $\operatorname{ker} R_{X}=\operatorname{ker} S_{X}=\operatorname{im}\left[\begin{array}{c}1 \\ -1\end{array}\right]$, then $X$ is also a solution of CGDARE $(\Sigma)$.

Suppose the initial and final states are constrained to be equal, i.e., $x(0)=x(T)$. Let $H=I_{2 n}, h_{0}=\left[\begin{array}{l}h_{1} \\ h_{2}\end{array}\right]$ and $h_{T}=0$. By taking $T=\left[\begin{array}{cc}1 & -1 \\ 1 & 1\end{array}\right]$, we obtain $T^{\mathrm{T}} R_{X} T=\operatorname{diag}\{4,0\}$, so that $R_{0, X}=4$. Moreover, $B T=\left[\begin{array}{cc}2 & -2 \\ 2 & 0\end{array}\right]$, so that $B_{1}=\left[\begin{array}{l}2 \\ 2\end{array}\right]$ and $B_{2}=\left[\begin{array}{c}-2 \\ 0\end{array}\right]$. Therefore, the reachable subspace of the pair $\left(A_{X}, B_{2}\right)$ is im $\left[\begin{array}{l}1 \\ 0\end{array}\right]$, which means this system is already in the desired basis. Thus, $A_{X, 11}=1, A_{X, 12}=A_{X, 22}=0$, $B_{11}=B_{12}=2$ and $B_{21}=-2$. In this case, (23), (26) and (27) become

$$
\begin{aligned}
x_{2}(t+1) & =B_{12} u_{2}(t), \\
\lambda_{2}(t) & =0 \cdot \lambda_{2}(t+1), \\
u_{2}(t) & =R_{X, 0}^{-1} B_{12}^{\mathrm{T}} \lambda_{2}(t+1) .
\end{aligned}
$$

This implies that

$$
\lambda_{2}(t)= \begin{cases}0 & t \in\{0, \ldots, T-1\} \\ \lambda_{2}(T) & t=T\end{cases}
$$

which leads to

$$
u_{2}(t)= \begin{cases}0 & t \in\{0, \ldots, T-2\} \\ R_{X, 0}^{-1} B_{12}^{\mathrm{T}} \lambda_{2}(T) & t=T-1,\end{cases}
$$

and

$x_{2}(t)= \begin{cases}x_{2}(0) & t=0 \\ 0 & t \in\{1, \ldots, T-1\} \\ B_{12}^{\mathrm{T}} R_{X, 0}^{-1} B_{12}^{\mathrm{T}} \lambda_{2}(T)=\lambda_{2}(T) & t=T .\end{cases}$

In this basis, (5) gives rise to $x_{1}(0)=x_{1}(T)$ and $x_{2}(0)=$ $x_{2}(T)=\lambda_{2}(T)$, which are linear in $x_{1}(T)$ and $\lambda_{2}(T)$, while (34) can be written as $x_{1}(0)+x_{1}(T)=h_{1}$ and $x_{2}(0)+x_{2}(T)+$ $\lambda_{2}(0)-\lambda_{2}(T)=h_{2}$. Since $\lambda_{2}(0)=0$ and $x_{2}(T)=\lambda_{2}(T)$, the latter can be written as $x_{2}(0)=h_{2}$. Therefore, the boundary conditions can be written in the form (35):

$$
\left[\begin{array}{cccc}
1 & 0 & -1 & 0 \\
0 & 1 & 0 & -1 \\
1 & 0 & 1 & 0 \\
0 & 1 & 0 & 0
\end{array}\right]\left[\begin{array}{c}
x_{1}(0) \\
x_{2}(0) \\
x_{1}(T) \\
\lambda_{2}(T)
\end{array}\right]=\left[\begin{array}{c}
0 \\
0 \\
h_{1} \\
h_{2}
\end{array}\right]
$$

This linear equation admits only the solution $x_{1}(0)=x_{1}(T)=$ $h_{1} / 2$ and $x_{2}(0)=\lambda_{2}(T)=h_{2}$. Now we can compute the optimal control law. First, $u_{2}(t)$ is zero for all $t \in\{0, \ldots, T-2\}$ and $u_{2}(T-1)=R_{X, 0}^{-1} B_{12}^{\mathrm{T}} \lambda_{2}(T)=h_{2} / 2$. In order to compute $u_{1}$, we write (20) as

$$
x_{1}(t+1)=1 \cdot x_{1}(t)-2 u_{1}(t)+\xi(t) .
$$

The term $\xi(t)$ in this case is equal to zero for all $t \in\{0, \ldots, T-2\}$ and $\xi(T-1)=B_{11} R_{0, X}^{-1} B_{12}^{\mathrm{T}} \lambda_{2}(T)=$ $\lambda_{2}(T)=h_{2}$. We can write (33) explicitly as

$$
\begin{aligned}
\lambda_{2}(T)= & h_{2} \text {. We can write (33) explicitly as } \\
x_{1}(T)= & x_{1}(0)+\left[\begin{array}{lllll}
I & A_{X, 11} & A_{X, 11}^{2} & \ldots & A_{X, 11}^{T-1}
\end{array}\right]\left[\begin{array}{c}
h_{2} \\
0 \\
\vdots \\
0
\end{array}\right] \\
& -2 \underbrace{\left[\begin{array}{llll}
1 & 1 & \ldots & 1
\end{array}\right]}_{T}\left[\begin{array}{c}
u_{1}(T-1) \\
u_{1}(T-2) \\
\vdots \\
u_{1}(0)
\end{array}\right]
\end{aligned}
$$

which gives

$$
\left[\begin{array}{c}
u_{1}(T-1) \\
u_{1}(T-2) \\
\vdots \\
u_{1}(0)
\end{array}\right]=\frac{h_{2}}{2 T}\left[\begin{array}{c}
1 \\
1 \\
\vdots \\
1
\end{array}\right]+\left[\begin{array}{cccc}
1-T & 0 & \ldots & 0 \\
1 & 2-T & \ldots & 0 \\
\vdots & \vdots & \ddots & \vdots \\
1 & 1 & \ldots & -1 \\
1 & 1 & \ldots & 1
\end{array}\right] v
$$

where $v$ is arbitrary and represents the degree of freedom in the control $u_{1}$. At this point it is easy to check that the trajectories generated with this control satisfy (20-25). If we choose for example $v$ to be equal to the second canonical 
basis vector of $\mathbb{R}^{T-1}$, using (36) we find

$$
\begin{aligned}
x_{1}(0)= & \frac{h_{1}}{2} \\
x_{1}(1)= & \frac{h_{1}}{2}-2\left(\frac{h_{2}}{2 T}+1\right) \\
\vdots & \\
x_{1}(T-2)= & \frac{h_{1}}{2}-2(T-2)\left(\frac{h_{2}}{2 T}+1\right) \\
x_{1}(T-1)= & \frac{h_{1}}{2}-2(T-2)\left(\frac{h_{2}}{2 T}+1\right)-2\left(\frac{h_{2}}{2 T}+2-T\right) \\
x_{1}(T)= & \frac{h_{1}}{2}-2(T-2)\left(\frac{h_{2}}{2 T}+1\right)-2\left(\frac{h_{2}}{2 T}-T+2\right) \\
& -2 \frac{h_{2}}{2 T}+\xi(T-1) .
\end{aligned}
$$

Since $\xi(T-1)=h_{2}$, the latter yields $x_{1}(T)=h_{1} / 2=x_{1}(0)$. This confirms that the optimality conditions are satisfied using these controls $u_{1}(t)$ and $u_{2}(t)$, which are therefore optimal.

\section{A. Existence of optimal solutions}

In general, the existence of a state trajectory $x(t)$ satisfying the constraints (1-2) for some $u(t)$ is not ensured, since we have not assumed reachability on (1). A necessary and sufficient condition for the existence of optimal solutions is that there exist state and input trajectories satisfying (1-2) (feasible solutions). In fact, since the optimal control problem formulated in Section II involves a finite number of variables - precisely, $L=m \cdot T$ for the control plus $n$ for the initial state - Problem 2.1 can be restated as a quadratic static optimization problem in these $L+n$ variables with linear constraints. Thus, a solution to Problem 2.1 exists if and only if a feasible solution - i.e., a state and input functions satisfying both (1) and (2) - exists.

Remark 5.1: The approach presented in this paper can successfully tackle even more general LQ problems, where the performance index is not necessarily positive semidefinite. E.g., consider

$$
\begin{aligned}
J(x, u)= & \sum_{t=0}^{T-1}\left[\begin{array}{ll}
x^{\mathrm{T}}(t) & u^{\mathrm{T}}(t)
\end{array}\right] \Pi\left[\begin{array}{l}
x(t) \\
u(t)
\end{array}\right] \\
& +x^{\mathrm{T}}(T) H x(T)+2 \zeta^{\mathrm{T}} x(T) .
\end{aligned}
$$

Although all the variational analysis remains unaffected, the presence of the term $2 \zeta x(T)$ deserves some considerations. Indeed, this linear term may cause the divergence to $-\infty$ of the cost index in correspondence to a sequence of admissible controls so that, even in the presence of feasible solutions, the optimal control may fail to exist. ${ }^{2}$ In this case, the linear equation representing the boundary conditions is infeasible. Two simple a priori sufficient conditions for the existence

\footnotetext{
${ }^{2}$ Consider for example the case where $A, B$ and $Q$ are the $2 \times 2$ identity matrices, while $R, S$ and $H$ are the zero matrices and $\zeta=\left[\begin{array}{ll}1 & 1\end{array}\right]^{\top}$. For this system, the LQ problem in one step (i.e., $T=1$ ) has no solution; in fact, the control $u(0)=-x(0)-m \zeta$ yields a value of the cost which goes to $-\infty$ as the parameter $m$ goes to $+\infty$.
}

of the optimal control and hence for the solvability of the two-point boundary-value problem are the following:

1) $\operatorname{ker} H \subseteq \operatorname{ker} \zeta^{\top}$. Under this condition, the cost on the final state (and hence the overall cost index) is bounded from below. Indeed, such a cost may be rewritten as a constant plus a positive semi-definite quadratic form $(x(T)-\bar{x})^{\top} H(x(T)-\bar{x})$ in the difference between $x(T)$ and a suitable "target state" $\bar{x}$. In this case the solution of the problem indeed exists.

2) $R>0$. In this case the current cost increases quadratically with the norm of the control input with the largest norm and, in the best situation, decreases linearly with the same norm. Thus the search for the optimal control input can be restricted to a compact set in $\mathbb{R}^{m \times T}$ and hence the optimal solution does exist.

\section{REFERENCES}

[1] A. Ferrante. On the structure of the solution of discrete-time algebraic Riccati equation with singular closed-loop matrix. IEEE Transactions on Automatic Control, AC-49(11):2049-2054, 2004.

[2] A. Ferrante, G. Marro, and L. Ntogramatzidis, "A parametrization of the solutions of the finite-horizon LQ problem with general cost and boundary conditions". Automatica, 41(8): 1359-1366, 2005.

[3] A. Ferrante and L. Ntogramatzidis. "Employing the algebraic Riccati equation for a parametrization of the solutions of the finite-horizon LQ problem: the discrete-time case". Syst. Control Lett., 54:693-703, 2005.

[4] A. Ferrante, and L. Ntogramatzidis, "A unified approach to finitehorizon generalized LQ optimal control problems for discrete-time systems". Linear Algebra Appl., 425(2-3):242-260, 2007.

[5] A. Ferrante, and L. Ntogramatzidis, "A Unified Approach to the FiniteHorizon Linear Quadratic Optimal Control Problem". Eur. J. Control, 13/5: 473-488, 2007.

[6] A. Ferrante, and L. Ntogramatzidis, "The Generalised Discrete Algebraic Riccati Equation in LQ optimal control". To appear in Automatica. Manuscript available at http: //arxiv.org/abs/1201.3704.

[7] A. Ferrante, and L. Ntogramatzidis, "Comments on Structural Invariant Subspaces of Singular Hamiltonian Systems and Nonrecursive Solutions of Finite-Horizon Optimal Control Problems", IEEE Trans. Aut. Control, 57(1):270-272, 2012.

[8] A. Ferrante, and H.K. Wimmer, "Order reduction of discrete-time algebraic Riccati equations with singular closed-loop matrix". Oper. Matrices, 1(1):61-70, 2007.

[9] V. Ionescu and C. Oară. Generalized discrete-time Riccati theory. SIAM J. Control Optim., 34(2):601-619, 1996.

[10] V. Ionescu, C. Oară, and M. Weiss. Generalized Riccati theory and robust control, a Popov function approach. Wiley, 1999.

[11] H. Kwakernaak and R. Sivan. Linear Optimal Control Systems. John Wiley \& Sons, New York, 1972.

[12] P. Lancaster and L. Rodman. Algebraic Riccati equations. Clarendon Press, Oxford, 1995.

[13] F.L. Lewis and V. Syrmos. Optimal Control. John Wiley \& Sons, New York, 1995.

[14] L. Ntogramatzidis, and G. Marro, "A parametrization of the solutions of the Hamiltonian system for stabilizable pairs". Int. J. Control, 78(7): 530-533, May 2005.

[15] A.A. Stoorvogel and A. Saberi. The discrete-time algebraic Riccati equation and linear matrix inequality. Linear Algebra Appl., 274:317365, 1998.

[16] M.A. Shayman. Geometry of the algebraic Riccati equation, Part I. SIAM J. Control Optim., 21:375-394, 1983.

[17] H. Trentelman, A. Stoorvogel, and M. Hautus, Control theory for linear systems, ser. Communications and Control Engineering. Great Britain: Springer, 2001. 